\title{
7 Things in history/things as history
}

Archaeology exists by virtue of 'things in history': it relies on things being part of history, changing as human behaviour or historical processes changed. Without this prerequisite, it would be impossible to reconstruct history based on objects. This book has placed things not only in history; it has also showed them as history, as themselves shaping historical trajectories. Terra sigillata became defined as a homogeneous and bounded category in production practices at Lezoux during the 2nd century AD. As a result, it became easier to compare sigillata pots one against another, which opened up prospects for competition. Comparability and competition in turn put up rigid standards for reproduction, resulting in a centralized production landscape with few and fairly large production sites. As a category, sigillata was fully defined by a limited number of traits (calcareous clays, oxidizing firing, shiny slips, etc.). Any one of these traits could be forwarded to fit the requirements of different stages in the distribution sequence (on board a ship, in a warehouse, etc.), and of different contexts of consumption (ritual, domestic, etc.). Nevertheless, the other traits were always mutually implicated (e.g. calcareous clays came with oxidizing firing), so that the different stages of the distribution sequence were made to hang together, or the different consumption contexts shared a material homogeneity that preceded meaning.

The historical trajectory thus shaped by the category of terra sigillata was not neutral. It fuelled a particular way of maintaining relations and channelling power; a way that resonates with the notion of 'empire' as centralized but wide-reaching, and as creating a structural homogeneity despite variable meanings and identities. Throughout this book, I have used active verbs such as 'shaped', 'facilitated', 'created' or 'constrained' to denote how sigillata impacted on history. Material culture was a causal force in history, but not in tune with templates of direct causation. A big challenge for material culture theory is to devise a more complex model of causality. This book coined the notion of trajectory as a first step towards getting to grips with material culture's causal historical role. The workings of a trajectory are eloquently described by Annemarie Mol and John Law.

'The point is not who has done it. Instead, what become more urgent are questions about what is happening. What do actors do? How are they creative? How do their underdetermined activities help to create or to destroy? What are the possibilities that they condition? Or, to speak as a walker in the Lake District hills: where does this path come from and where might it lead? ${ }^{762}$

A thing's trajectory is not the same as an artefact's biography. ${ }^{763}$ The crucial difference is that a trajectory plots the generic possibilities of a kind of thing (in this case, the category of terra sigillata, or the rooted 'Rhenish' wares), not the actual events happening to a specific artefact. In that sense, trajectories are not unlike Gosden's 'genealogies' that show how '[p]atterns of exchange or consumption derive partly from the nature of the objects themselves'. ${ }^{764}$

The notion of things being defined contextually, and therefore being redefined as they pass through different contexts was already central to biographical approaches and plays a key role in this study. With biographical approaches, however, the link between these different stages of redefinition is accounted for solely by human practices and choices. The shape of the biography is incidental to the object itself

762 Law/Mol 2008, 74.

763 Appadurai 1986; Gosden/Marshall 1999; Hoskins 2006; Foster 2006; Kopytoff 1986.
764 Gosden 2005, 196. Gosden however focuses on 'style', as per Gell 1998. Compare to Olsen et al. 2012, 194, $170-171$. 
that was gifted, traded, or passed around. What material agency does for the framework of trajectories expounded in this book is to provide a non-trivial link between the different stages in a chain of redefinition. By shaping the possibilities for action at each stage, material agency always extends forwards in time. As a result, the historical logic is in the trajectory, and no longer merely impinging on it from the outside, as was the case with biographies.

To be clear, this material agency residing in the links making up trajectories is not on a par with the criteria of 'commonsensical' human-like agency. Most importantly, it does not imply intentionality. Instead of intentionality, however, there is a loose sense of directionality. With a twist to phenomenological speak, things with a certain kind of material agency are preferentially directed to certain kinds of actions: in consumption, for instance, the categoryness of terra sigillata facilitated widespread integration, whereas Trier 'Rhenish' wares were predisposed through their rootedness to special kinds of uses. Such directedness suggests a model of causation in which 'there might exist many metaphysical shades between full causality and sheer inexistence'. ${ }^{765}$

Thinking in terms of different kinds of material agency, each developing different trajectories, significantly adds to the various conceptual studies of material agency with which this book started. ${ }^{766}$ The question is no longer 'is material culture active or not?', but 'how is it active?'. The analyses presented here walk the line between generalized theoretical musings on the workings of material culture and idiosyncratic case studies of a particular artefact (as in biographical approaches). In the shape of trajectories, material agency transcends contextual specificity, yet remains indicative of historical processes. Such a mid-level scale provides scope for comparative study, for instance comparing 'categories' across time and space, as in the analogy between terrra sigillata pottery and Coca-Cola.

This strikes a chord with a recent comparative trend in classical archaeology, not in the least focusing on empires ${ }^{767}$ The model of trajectories promises important finetuning of current comparative studies, most importantly because of its adjusted model of causality. As a result, comparison is no longer a matter of isolating cross-context similarities and attributing them to 'bigger' processes (typically climate), while the differences are relegated to 'historical specificities'. Comparison between Coca-Cola and terra sigillata pottery as categories does not proceeed from a checklist of similarities and differences, but identifies processes and possibilities, such as standardisation, comparability, cross-context consumption, etc. It does not claim to come with conclusions about climate, economic organisation, or other big external invariables, but it does get at something over and above the specificity of modern capitalism or the Roman empire.

The main point is that recognising how things shaped historical trajectories leads to a better use of material culture in historical narratives. This is graphically illustrated in figure 7.1. A retrospective model of material culture, starting from sigillata as a homogeneous and wide-spread category, is bound to search for causal explanations in external actors (grey in Fig. 7.1). As a 'ready-made' type, sigillata was forced in production based on economic considerations by traders and investors, its production knowledge was carried around in the heads of migrating craftsmen, its consumption a choice of identity-building consumers. Through their actions and decisions, these external actors then affected historical developments, such as economic growth or cultural change. The main research question is one of 'who'. But this is generally not a question that archaeology is equipped to answer. The unfortunate result is that material culture - and by extension archaeology - is underused in historical narratives, in particular of the Roman period. Archaeological evidence tends to come in only in a second stage, to prove or disprove models developed on the basis of other evidence (e.g. price lists for economic history ${ }^{768}$ ).

765 Latour 2005, 72.

766 Cf. Foucault 1986; Latour 2012 on different modes of existence; Law/Mol 2001; Mol/Law 1994; Van Oyen 2015 c.
767 E.g.Vasunia 2011.

768 Bowman/Wilson 2009. 


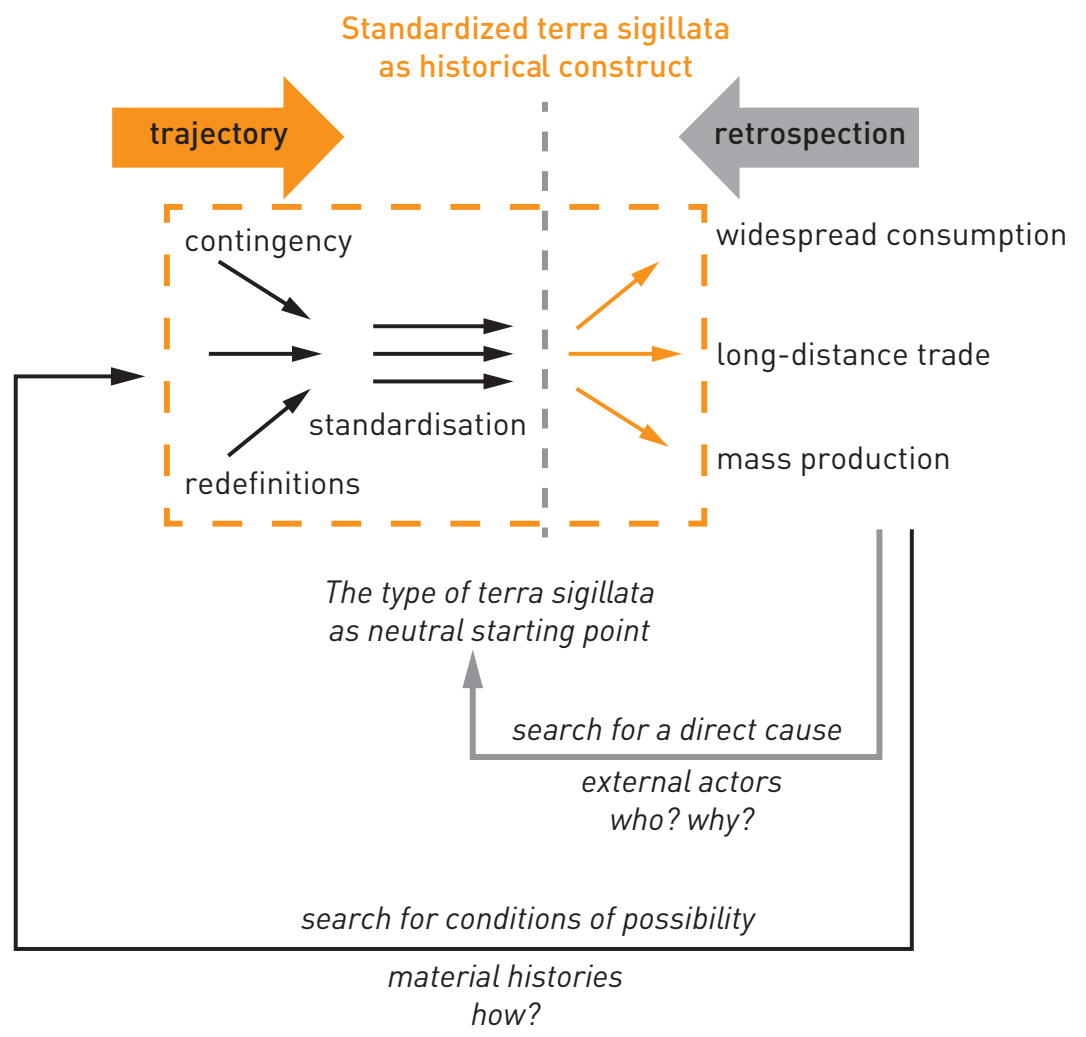

Fig. 7.1. Retrospection versus trajectories.

The research question throughout this book has been 'how' instead of 'who' (black in Fig. 7.1). How are things defined, and how do they shape the range of possible actions? This is a question that archaeology is actually very good at answering. Chaine opératoire approaches have long described the 'how' of production sequences (how clays are prepared, how pots are fired), and contextual study details the 'how' of consumption practices (how different artefacts relate to each other, how pots were used). But the notion of trajectory - of things as history-makers - lends historical impact to the question of 'how'. Description ('how') is no longer separated from explanation ('why'); it becomes a necessary prerequisite for it.

Nevertheless, 'material histories' on the model of this book are not incompatible with more traditional histories. ${ }^{769}$ Throughout the preceding chapters, emphasis was placed on how a non-retrospective approach contributes to key questions relying on 'things in history'. In addition, it helps contextualize the tools and questions of traditional histories. We can now state, for instance, that it does not make sense to include terra sigillata and 'Rhenish' ware pots in a single graph to examine economic growth: the same triggers did not result in comparable empirical patterns in either case. For example, investment resulted in long-distance trade and quantitative increase in production for sigillata versus selective regional focus and limited niche in consumption for 'Rhenish' wares. This difference in trajectory cannot be read off from a mere quantitative table or a simple bar chart.

At the same time, big questions such as empire also get reframed as trajectories for which the defining question is 'how', not 'who' or 'why'. As discussed in the conclusion to the preceding chapter, empire becomes the cumulative effect of the trajectory of (terra sigillata as a) category (red in Fig. 7.1). This effect consisted of a centralized structure, reaching widely, in a recognizable but semantically open material environment. Empire therefore is no longer a causal chimera in and of itself, but is rooted deep in the possibilities for action of some of its signature material culture, like terra sigillata pottery for the Roman

769 Stahl 2010 for the term 'material histories'. 
empire. Empire was formed as terra sigillata pots were made - the one did not precede the other. And empire was no more or less stable or all-encompassing than was terra sigillata's categoryness: it relied on a continuous process of material and conceptual Othering, and its trajectory was paired with for instance rooted 'Rhenish' wares and their very different possibilities for action.

Because of their cumulative nature, material histories do not have a zero point, contrary to the longstanding archaeological fascination with origins. The history presented here sketches the material conditions and possibilities of empire in its (relatively) stable form; it does not touch on the earlier phases of imperialism. This was a necessary analytical move to do away with the retrospective approach to material culture, in which terra sigillata is always 'already made': even in this apparently stable period, terra sigillata was always becoming, being made and actively stabilized, both practically and conceptually. But in theory, trajectories can start and end anywhere and anytime. ${ }^{770}$ Their analytical decentralisation shares the concerns of recent post-colonial and globalisation studies with the old centralized, top-down, elite-focused Romanization models. ${ }^{71}$ If this book would have traced terra sigillata production from Central Gaul to the Argonne area instead of to Trier, the resulting trajectories may well have had a different shape. Nevertheless, those alternative material histories would be historically related, because of the model of causality (as setting conditions of possibility) on which trajectories build. Different to recent 'decentralized' narratives, however, analytical decentralisation need not dictate interpretive decentralization. Based on a decentralized analytical framework, then, this study could still make a case for the importance of centralisation in the trajectory of terra sigillata and the related material history of the Roman empire. But such processes of centralisation then become emergent in nature.

Material histories thus build on descriptive trajectories. But the way in which sigillata is processed and studied sets limits to the kinds of descriptions possible, and thus also feeds into the historical explanations we can come up with. ${ }^{772}$ This has several important repercussions for archaeological practice. In order to harness their explanatory potential, the descriptions in this book had to go into the detail of terra sigillata, discussing clays, the physical arrangement of production sites, or the contents and organisation of a ship's cargo. In current academic practice, however, the institutional structure is such that detailed, specialist artefact studies are kept separate from grand historical narratives. The idea is that one can talk about the latter by merely skimming over or summarizing the former, as if history proceeds on nested scales of abstraction. Historical narratives about the culture or economy of the (western) Roman world are published as monographs, while specialist artefact studies are hidden away in grey literature or site catalogues. Conferences will be either about big questions, such as 'Romanization' or 'economic growth', or they will discuss the minutiae of new pottery typologies and production sites. The institutional pathways are laid in such a way that description is forced to remain 'mere description'. A new, non-retrospective model of material culture as developed in this book can go part of the way towards mediating this, but will have to be met halfway by critical reflection on and change of such institutional pathways.

What stands out most perhaps at the end of this book is the resonance between how sigillata is maintained as a separate, homogenous category in present practices of study (Chapter 2), and how it became defined as a category in practices of production, distribution and consumption in Roman times (Chapters 3-6). Typologies, specialisation, and division of site reports by artefact class work remarkably well for processing sigillata precisely because sigillata pottery was indeed defined as a separate category with a limited package of traits in the past. Cutting through the different case studies in this book, however, were things that did not conform to this template: black-gloss wares, Lezoux 'Rhenish' wares as sigillata's Other, or the rooted Trier 'Rhenish' wares. Still, current practices of study impose the same typologies, specialisation, and division of site reports on those artefact classes. This is not to say that for instance typologies cannot be useful in these cases - they can play an important role in pointing out incongru-

770 Cf. Strathern 1996.

771 Mattingly 2006; Pitts/Versluys 2015; Versluys 2014; J.
Webster 2001.

772 Cf. Gosden 2010. 
ences. It is indeed clear that the many attempts at pinning down identifying traits for 'Rhenish' wares are in vain or partial. But noting such incongruences cannot be the end point, and we somehow need to alter our practices of study or keep them flexible enough to accommodate things that were defined by different parameters and to avoid neglecting or misrepresenting them. Conversely, typologies and similar tools for analysis can no longer be considered as neutral mechanisms for organising data; they also say something about things' historical role.

This book has taken on the challenge of linking the insight that material culture is active with largescale historical narratives. It has shown how terra sigillata, Roman archaeology's most emblematic type of pottery, itself facilitated its widespread and dense archaeological pattern, and led the way towards specific cultural and economic processes. The result is a truly archaeological or material history, not just telling us about things in history, but about things as history. The analytical trick to achieve this result has been not to approach material culture retrospectively, but to follow its emergence and historical trajectory. Although building on a long history of terra sigillata scholarship and material culture studies, this book aims to be a starting point for research, not an end point. Future work will need to expand the nonretrospective analytical trick to other material, periods, and regions, and couple it with actual changes in our practices of study. 
